\title{
普洱茶减肥、降脂机制的探讨
}

\author{
邹晓菊 ${ }^{1}$, 丁毅弘 ${ }^{2}$, 梁 斌 ${ }^{2, *}$ \\ (1. 昆明学院 生命科学与技术系, 云南 昆明 $650214 ; 2$. 中国科学院和云南省动物模型与人类疾病机理重点实验室, \\ 中国科学院昆明动物研究所, 云南 昆明 650223)
}

\begin{abstract}
摘要: 普洱茶(Pu-erh tea)产于云南省南部地区, 具有悠久的发展历史和丰富的文化涵蕴, 被认为具有减肥、降 脂、降血糖、防止心脑血管疾病等代谢性疾病功效。近几年来在人、啮齿类和细胞水平的研究表明, 普洱茶的确 具有减肥、降脂功效，普洱茶中的茶色素、多糖、多酚和他汀类物质可能通过抑制脂肪合成、促进脂肪氧化分解 而发挥其减肥、降脂功效。本文综述了目前关于普洱茶减肥、降脂的生物学功效、活性成分、以及作用机制的相 关报道，同时探讨了该领域未来的一些研究方向。
\end{abstract}

关键词: 普洱茶; 减肥、降脂; 机制; 活性成分

中图分类号：Q946 文献标志码：A 文章编号：0254-5853-(2012)04-0421-06

\section{The mechanisms of weight-cutting effect in Pu-erh tea}

\author{
ZOU Xiao-Jü ${ }^{1}$, DING Yi-Hong ${ }^{2}$, LIANG Bin ${ }^{2, *}$
}

(1. Department of Life Science and Biotechnology, Kunming University, Kunming 650214, China; 2. Key Laboratory of Animal Models and Human Disease Mechanisms of the Chinese Academy of Science \& Yunnan Province, Kunming Institute of Zoology, Kunming 650223, China)

\begin{abstract}
Originally grown and produced in southern of Yunnan, China, Pu-erh tea has a long history and carries rich cultural connotations. Consumption of Pu-erh has been thought to possess numerous health benefits including weight-loss, lowering of blood glucose levels, and preventing cardiovascular diseases. Research on humans, rodents, and cell lines have each confirmed that Pu-erh tea indeed displays weight-loss and blood lipid lowering effects. The main bioactive components, such as theabrownin (TB), polysaccharides, polyphenols, and statins, may down-regulate the biosynthesis of fat and up-regulate the oxidation of fat to cut weight and reduce the content of lipids in blood. Here, we summarize current progress on understanding the mechanisms and bioactive components of Pu-erh's weight-cutting effects as well as highlighting current weaknesses in the field in order to suggest possible solutions for future research on Pu-erh tea.
\end{abstract}

Key words: Pu-erh tea; Weight-cut effect; Mechanisms; Bioactive components

肥胖是由于能量摄入超过消耗，多余能量以脂 肪形式存储，导致脂肪细胞体积增大和过度增殖的 代谢性疾病。肥胖症不仅在发达国家很普遍，在我 国也随着人口饮食结构和生活方式的变化, 呈快速 上升趋势。目前全世界大约有 10 亿人体重超重, 其 中 $2 / 3$ 属于肥胖。据卫生部和世界卫生组织 (WHO) 2011 年联合报道, 我国是目前全世界肥胖增 长率最高的国家，1992-2002 年，中国人超重和肥 胖增长率分别为 $38 \%$ (3.05 亿)和 $81 \%(1.2$ 亿)。肥胖
和糖尿病、心脑血管疾病、高血压、中风、非酒精 性脂肪肝、肿瘤等人类重大代谢性疾病高度相关。 因此，寻找和开发具有减肥、降脂功效的药物，特 别是传统中医药, 是有效防治肥胖及相关代谢性疾 病的重要策略和途径。

普洱茶原产于我国云南西双版纳及普洱一带, 是以云南特有的大叶种晒青毛茶在高温高湿的环 境中以及微生物的参与下, 经特殊后发酵工艺包括 自然发酵(非酶性氧化)和渥堆发酵(微生物酶促氧

收稿日期: 2012-04-05; 接受日期: 2012-04-20

基金项目：国家自然科学基金地区基金(31160216); 国家自然科学基金面上项目(31171134); 云南省应用基础研究计划项目（2011FZ179); 昆明 学院校级研究项目 (YJL11018)

*通讯作者(Corresponding author), 梁斌, 博士, 研究员。Email: liangb@mail.kiz.ac.cn 
化)而制成的发酵茶。普洱生茶为未经发酵的晒青毛 茶; 普洱熟茶为采用渥堆工艺, 经微生物发酵 后, 加工形成的散茶和紧压茶。普洱熟茶保存可 长达 $20 \sim 50$ 年, 甚至更久。普洱茶的减肥、降脂、 降血糖、防治心脑血管疾病、抗衰老、抗肿瘤、抗 病菌、抗病毒等多种保健功能及其作用机理研究主 要针对普洱熟茶, 且相关研究已开展近 20 年。本文 综述了 PubMed 和中国知网( “中国期刊全文数据 库”、“中国博士学位论文全文数据库” 和 “中国优 秀硕士学位论文全文数据库” 等三个数据库)中关于 普洱茶减肥、降脂的文献; 归纳了普洱茶减肥、降 脂的生物学功效、主要活性成分和作用机理的相关 报道; 并探讨了该研究领域中现存的不足, 以及未 来的方向和策略。

\section{1 普洱茶减肥、降脂功效}

普洱茶在东亚、东南亚和欧美国家享有“美容 茶”、“减肥茶”和“窈察茶”等美称。Fujita \& Yamagami (2008)对高胆固醇血症患者进行了 4 个 月连续摄入普洱茶提取物的临床实验, 发现患者体 重及血液中总胆固醇(TC)和低密度脂蛋白（LDL） 水平均显著降低, 且无其他生化指标的改变。 $\mathrm{Li}$ et al (2009) 在给 60 例血脂异常患者服用普洱茶片后, 发现患者的血液总胆固醇(TC)、甘油三脂(TG)、低 密度脂蛋白 (LDL) 均显著降低。

大多数关于普洱茶减肥、降脂功效的研究是在 啮齿动物(大、小鼠)和细胞系上进行的。饲喂普洱 茶能降低实验大鼠体重及腹部脂肪组织重量, 降低 其血清总胆固醇(TC)、甘油三酯(TG)及低密度脂蛋 白(LDL)含量, 增加高密度脂蛋白(HDL)含量及减 缓肝组织脂肪变性 (Sano et al,1986; Yang \& Koo,1997; Huang \& Lin, 2011; Kuo et al, 2005; Cao et al, 2011; Gong et al, 2010; Hou et al, 2009; Huang et al, 2010; Jiang \& Shao, 2011; Wang et al, 2011; Wu, 2005; Xu et al, 2011; Yuan et al, 2009; Zhao \& Shao, 2010); 人肝癌 HepG2 细胞实验也证实了普洱茶的 减肥、降脂功效(Chiang et al, 2005; Way et al, 2009)。

Kuo et al (2005)饲喂大鼠绿茶、乌龙茶、红茶 及普洱茶 30 周后, 发现其减肥功效顺序为乌龙茶> 普洱茶>红茶>绿茶; 普洱茶能在提高高密度脂蛋白 (HDL) 含量的同时降低低密度脂蛋白(LDL)含量, 而其它茶则同时降低两者含量; 相对于乌龙茶和红 茶, 普洱茶和绿茶能更有效地降低血液总胆固醇
(TC), 提示普洱茶的减肥、降脂功效可能较其他茶 更为明显。

\section{2 普洱茶减肥、降脂活性成分}

普洱茶的主要活性成分和其他茶一样, 为茶多 酚(polyphenols)、茶多糖(polysaccharides)、茶色素 (tea pigments) 和他汀类物质 (statins) 等 ( $\mathrm{Gu}$ et al, 2011)。高温高湿的渥堆过程中，在微生物的作用下， 茶多酚发生的氧化、降解、缩合等化学反应将形成 化学结构更为复杂的酚类成分; 茶多酚没食子儿茶 素没食子酸 (epigallocatechin-3-gallate, EGCG)、没 食子儿茶素(epigallocatechin, EGC), 儿茶素没食子 酸 (epicatechin-3-gallate, ECG)含量下降; 没食子酸 (gallic acid, GA)、茶多糖和茶色素含量增高( Ku et al, 2010; Gong et al, 2010; Wang et al, 2011); 以儿茶素 为主的多酚类化合物在渥堆过程中氧化衍生出的 水溶性色素混合物, 即茶色素, 可分为茶黄素 (theaflavins, TFs)、茶红素(thearubigins, TRs)、茶褐 素(theabrownins, TBs), 其含量依次递增(Luo et al, 1998); 另外, 发酵过程中, 一些微生物也能产生具 有降脂功能的他汀类物质(Jeng et al, 2007; Yang et al, 2007)。

普洱茶的复杂成分中, 主要有以下几类活性物 质在发挥减肥、降脂的作用。

\section{1 茶褐素}

茶褐素是一类溶于水而不溶于乙酸乙酯和正 丁醇的复杂褐色色素化合物, 除含有多酚类氧化聚 合产物外, 还含有氨基酸、多糖等结合物(Shao et al, 2011), 是普洱茶的主要活性成份(Wang et al, 2011), 含量为 $100 \sim 140 \mathrm{~g} / \mathrm{kg}$ 。普洱茶中茶褐素的形成机制 尚不清楚, Wang et al (2011)发现, 微生物中的酶是 普洱茶发酵过程中茶褐素形成的主要原因。Chen et al (2011)和 Gong et al (2010)的研究分别揭示普洱茶 茶褐素可显著降低高脂血症大鼠血清中总胆固醇 (TC)、甘油三脂(TG)及低密度脂蛋白(LDL)水平, 升 高高密度脂蛋白(HDL)水平, 并减少大鼠肝脏脂肪 沉积, 预防脂肪肝形成。Gao et al (2010)和 Gong et al (2010)也分别报道普洱茶茶褐素可显著增强大鼠 肝脏和附睪脂肪组织激素敏感性脂肪酶 (hormone-sensitive lipase, HSL)的活性及其 mRNA 的表达, 具有明显的降血脂功效。以上研究提示茶 褐素可能是普洱茶中的重要的减肥、降脂成分, 但 由于茶褐素分子量极大、组成复杂, 因此对其具体 
结构以及作用机理的研究目前还几乎是空白。

\section{2 茶多糖}

茶多糖(TPS) 由糖类、蛋白质、果胶和灰分等 组成, 是一类与蛋白质结合的酸性多糖或酸性糖蛋 白, 具有降血糖、降血脂、提高机体免疫力及抗氧 化等保健功能。Wu \& Wu (2006)的研究表明茶多糖 剂量与小鼠血清低密度脂蛋白(LDL)含量的下降及 高密度脂蛋白(HDL)含量的上升呈正比, 即茶多糖 对高脂饮食小鼠的血脂升高存在量效抑制作用。Wu et al (2007)发现与高脂对照组小鼠相比, 茶多糖组 处理小鼠血清中甘油三脂(TG)、总胆固醇(TC)和低 密度脂蛋白(LDL)水平分别下降了 $40.60 \% 、 25.36 \%$ 和 $24.38 \%$, 而高密度脂蛋白 (HDL) 水平上升了 $73.44 \%$ 。 Hou et al (2008)的研究表明茶多糖可降低 高血脂大鼠总胆固醇(TC)和丙二醛(MAD)含量, 增 加高密度脂蛋白(HDL) 含量, 但对低密度脂蛋白 (LDL)含量无显著影响。

\section{3 茶多酚}

茶多酚是从茶叶中分离提取的多酚类化合物 复合体，可降低外周血甘油三酯(TG)、总胆固醇 (TC)、低密度脂蛋白胆固醇(LDL)水平, 升高高密度 脂蛋白胆固醇(HDL)水平, 具有防治高血脂症的作 用，且对载脂蛋白具有一定的调节作用，另外，茶 多酚还可以增加血超氧化物歧化酶(SOD)活性及抗 脂质的过氧化(Chen \& Guo, 2006)。

由于普洱茶在后发酵过程中，以茶多酚为主的 多酚类成分大量氧化衍生成为茶色素, 儿茶酚含量 下降，而没食子酸(GA)和咖啡因的含量提高(Zhang et al, 2011), 从而造成普洱茶中茶多酚降血脂的功 效不明显(Wu et al, 2007)。没食子酸(GA)是没食子 儿茶素没食子酸(EGCG)的主要降解产物, 具有抗 菌、抗炎、抗肿瘤、抗突变等作用(Ow \& Stupans, 2003)，且能抑制 HepG2 细胞的胆固醇合成( $\mathrm{Lu} \mathrm{\&}$ Sun, 2006)。因此, 高含量没食子酸可能是普洱茶保 健功能的重要因素(Lu et al, 2007)。

\section{4 他汀类物质}

3-羟基-3-甲基戊二酰辅酶 $\mathrm{A}$ 还原酶(3-hydroxy3-methylglutary coenzyme A reductase, HMGR)是胆 固醇生物合成的第一步限速酶。他汀类物质, 如洛 伐他汀(lovastatin)、辛伐他汀 (simvastatin)是 HMGR 的抑制物。因此，这些抑制剂可以降低胆固醇含量, 降低心、脑血管疾病发病几率。他汀类物质具有内 酯结构(lactone)和羟基酸结构 (hydroxy acid)。在体
内, 羟基酸结构是活性结构, 能降低血液中的胆固 醇; 而内酯结构的他汀物质能被肠道吸收, 在肝脏 和其他组织转化为活性羟基酸结构。 Yang et al (2007)发现云南普洱茶中含有以内酯结构存在的洛 伐他汀，而沸水 $\left(100{ }^{\circ} \mathrm{C}\right)$ 能把普洱茶中无活性的内 酯结构洛伐他汀转为活性羟基酸结构。同时, Jeng et al (2007)发现普洱茶储存的年代越久, 含有的他汀 类物质含量越高。这些研究提示普洱茶中他汀物质 的减肥、降脂作用。

\section{3 普洱茶的减肥、降脂机制}

肥胖是遗传和环境因素共同作用所致的综合 代谢性疾病。减肥药物主要通过减少食物摄入和吸 收，促进食物排泄，抑制脂肪合成，加快脂肪分解 起作用。

Zhou et al (2009)发现普洱茶可明显抑制小肠 对木糖的吸收，并增加脂肪排出量。脂肪合成酶 (fatty acid synthase, Fas)每次增加两个碳原子，延伸 脂肪酸, 是脂肪合成的关键酶之一。普洱茶能抑制 大鼠肝脏及人肝癌 HepG2 细胞的脂肪合成酶 mRNA 和蛋白水平(Chiang et al, 2005; Huang \& Lin, 2011)。普洱茶可能作用于 PI3K、JNK、P38 或 MEK 等激酶(Chiang et al, 2005), 提高 AMP 活化蛋白激 酶(AMPK)的磷酸化水平，进而抑制脂肪合成酶表 达 (Huang \& Lin，2011)。普洱茶可能通过抑制 PI3K/Akt 和 JNK 信号通路(Chiang et al, 2005)，或者 通过 LKB1 通路激活 $\mathrm{AMPK}$ 来降低大鼠肝脏内脂肪 酸合成酶的表达和乙酰辅酶 $\mathrm{A}$ 羧化酶 (acetyl-coenzyme A carboxylase, ACC)的活性，抑制 脂肪合成(Way et al, 2009)。

固醇调节元件结合蛋白-1c(sterol regulatory element binding protein-1c, SREBP-1c)是调控脂肪 合成的重要转录因子，它调控编码脂肪合成酶、乙 酰辅酶 $\mathrm{A}$ 羒化酶和硬脂酰去饱合酶(stearoyl CoA desaturase 1, SCD1)等基因的表达。Yuan et al (2009) 发现普洱茶下调 SREBP-1c、SCD1、以及参与甘油 三脂合成最后一步的酶二酰基甘油酰转移酶 -1(diglyceride acyltransferase, DGAT1)等基因的表达, 从而减少脂肪合成。Way et al(2009)比较了不同方法 提取的普洱茶减肥效果, 发现乙酸乙酯提取的普洱 茶 PR-3-5s 组分能降低肝癌细胞 HepG2 高脂含量, 它通过 LKB1 通路刺激 AMPK 而抑制脂肪合成酶表 达、以及乙酰辅酶 $\mathrm{A}$ 羧化酶活性。 
普洱茶也可能通过上调脂肪氧化、分解达到减 肥、降脂功效。Zhang et al (2009)发现普洱茶醇提取 物对调控脂肪氧化的 PPAR $\gamma$ 受体有激活作用, 其 活性成分很可能是没食子酸。Huang et al (2010)检 测饲喂普洱茶小鼠的肾上腺素受体表达量和体重 变化, 推测普洱茶可能通过咖啡碱的作用, 调高肾 上腺素受体表达水平, 刺激脂肪氧化、分解达到减 肥目的。激素敏感性脂肪酶(HSL)是在脂肪细胞内 分解甘油三脂的脂肪酶, 是动物脂肪分解代谢的限 速酶之一。Cao et al (2011), Gao et al (2010)和 Gong et al (2010)分别报道了普洱茶中肝脏肝脂酶(hepatic lipase, HL)和激素敏感性脂酶的表达和活性上升可 促进脂肪分解。

总之, 这些研究揭示了普洱茶很可能是通过减 少能量摄取, 增加脂肪排出, 抑制脂肪合成, 促进 脂肪氧化、分解, 以及调节相关代谢信号通路的作 用来达到减肥、降脂效果(图 1)。

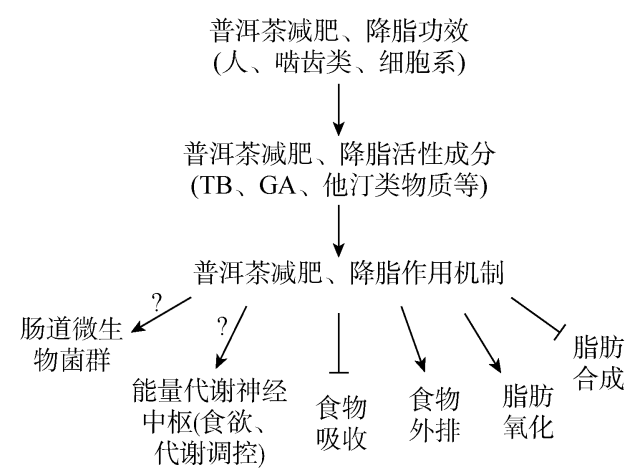

Fig. 1 Model of the weight-cutting mechanisms and bioactive components in Pu-erh tea

$\rightarrow$ : 激活或正调控; †：抑制或负调控。

$\rightarrow$ : indicates activation or positive regulation; $-\dashv:$ indicates inhibition or negative regulation.

\section{4 现存问题和未来研究展望}

普洱茶广受海内、外欢迎的一个重要原因为其 减肥、降脂、防治心血管疾病病、降血糖等保健功 效, 然而, 其保健功能的相关研究却严重滞后。未 来的研究需要从以下几个方面来加强。

\section{1 不同普洱茶产品的减肥、降脂功效比较}

普洱茶由于茶树品系、产地的不同、以及采后 发酵处理的差别, 形成多种普洱茶产品, 但这些产 品缺乏明确的质量标准和公认的品牌。这不仅制约 了普洱茶的产业发展，也阻碍对普洱茶功效、活性 成分和作用机理的深入研究。对此，未来应该大力 开展不同普洱茶产品功效的比较研究。寻找和确认
具有良好减肥、降脂功效的产品。

\section{2 加强普洱茶减肥、降脂功效的临床研究}

大多数关于普洱茶减肥、降脂的研究是在大、 小鼠和细胞系水平进行的, 而以人为研究对象的临 床研究却甚少，且病例少、时间短、实验设计过于 简单，结果缺乏说服力。因此，未来需加强普洱茶 的临床研究。普洱茶具有上千年的历史, 不少人终 身饮用。因此，可以大规模调查、比较长期习惯饮 用普洱茶人群和对照人群(无饮用普洱茶、其它茶, 或者咖啡习惯)的健康状况。同时，可以开展健康人 群与肥胖人群(尤其是具有肥胖相关代谢性疾病人 群)的短期(几周)到长期(几年)饮用普洱茶后的健康 状况研究, 重点检测体重、血液、尿液代谢变化等。

\section{3 普洱茶减肥、降脂活性成分的确定}

普洱茶含有的茶多酚、茶多糖、茶褐素、氨基 酸、咖啡因及他汀类物质等多种成分均具有减肥、 降脂功能。目前尚不明确其中哪种成分单独或者和 其他成分共同起减肥、降脂作用，或者还有其他未 知化合物成分起作用。

对茶叶中减肥、降脂成分的研究主要集中在茶 多酚和茶多糖上。近几年来, 利用液相色谱/质谱方 法对普洱茶成分和发酵过程中各种成分变化的研 究取得了不少进展( Ku et al, 2010; Qin et al, 2012; Syu et al, 2008; Wang et al, 2011; Xie et al, 2009; Zhang et al, 2011; Zuo et al, 2002), 这些研究表明, 茶褐素(TB) 和没食子酸 $(\mathrm{GA})$ 是普洱茶的主要成分。 目前, 至少有 3 个研究组报道了茶褐素的减肥、降 脂功效(Chen et al, 2010; Gao et al, 2010; Gong et al, 2010), 暗示普洱茶的主要作用成分可能是茶褐素 (TB)。然而，茶褐素是一类十分复杂的化合物，除 含有多酚类氧化聚合产物外，还含有氨基酸、多糖 等结合物。对普洱茶茶褐素组成和功能的研究急待 加强和提高。没食子酸 $(\mathrm{GA})$ 也是普洱茶的主要成分, 它是否和茶褐素具有同样的减肥、降脂功效，或者 与茶褐素混合使用时效果更明显? 普洱茶中的氨 基酸、咖啡因和他汀类物质等成分含量较低，但它 们单独或者和茶褐素 $(\mathrm{TB})$ 、没食子酸 $(\mathrm{GA})$ 等按一定 比例配比混合后，是否会提高普洱茶的减肥、降脂 功效? 这些研究目前几乎是空白。因此, 未来需要 发展更好的方法和技术以全面了解普洱茶产品的 成分、化合物含量、组成和结构等; 另外更需要在 细胞和动物模型水平比较普洱茶中具有减肥、降脂 功效成分的单独及混合效果。 
4.4 多方面、系统性探索普洱茶减肥、降脂作用 机理

虽然普洱茶减肥、降脂的作用机理研究已取得 不少进展, 但大多数研究缺乏系统性, 往往只注重 于参与脂代谢的某一个基因和信号通路。

脂代谢及其调控机制受遗传和环境因素影响。 目前尚不清楚普洱茶是否也作用于神经信号, 如: 下丘脑食欲和能量调控中枢, 以降低食欲并减少食 物吸收和利用(图 1)。近几年的研究发现, 人肠道微 生物菌群的差异是导致肥胖的重要因素(Turnbaugh \& Gordon, 2009)。普洱茶是否也能改变人肠道微生 物菌群呢(图 1)?

我们采用系统生物学方法, 在人、和大、小鼠 中分别找到了 356、392、380 个脂类代谢基因。另 外，目前已经报道人和大、小鼠基因组中大约有

\section{参考文献:}

Cao ZH, Gu DH, Lin QY, Xu ZQ, Huang QC, Rao H, Liu EW, Jia JJ, Ge CR. 2011. Effect of pu-erh tea on body fat and lipid profiles in rats with diet-induced obesity[J]. Phytother Res, 25(2): 234-238.

Chen P, Wang XQ, Song WJ. 2011. Weight-reducing effect of pigment from Pu-erh tea on Diet-induced obese rats[J]. Food Res Dev, 32(7): 169-171. [陈沛, 王雪青, 宋文军. 2011. 普洱茶茶色素减肥作用的 研究. 食品研究与开发, 32(7): 169-171.]

Chen YS, Liu BL, Chang YN. 2010. Bioactivities and sensory evaluation of $\mathrm{Pu}$-erh teas made from three tea leaves in an improved pile fermentation process[J]. J Biosci Bioeng, 109(6): 557-563.

Chen YX, Guo CJ. 2006. The research progress on Hypolipidemic effect of tea polyphenols and its mechanism[J]. Food Nut China, (4): 47-49. [陈 玉霞, 郭长江. 2006. 茶多酚降血. 脂作用及其机制研究进展. 中国 食物与营养, (4): 47-49.]

Chiang CT, Weng MS, Lin-Shiau SY, Kuo KL, Tsai YJ, Lin JK. 2005. $\mathrm{Pu}$-erh tea supplementation suppresses fatty acid synthase expression in the rat liver through downregulating Akt and JNK signalings as demonstrated in human hepatoma HepG2 cells[J]. Oncol Res, 16(3): 119-128.

Fujita H, Yamagami T. 2008. Efficacy and safety of Chinese black tea (Pu-ehr) extract in healthy and hypercholesterolemic subjects[J]. Ann Nutr Metab, 53(1): 33-42.

Gao B, Peng CX, Gong JS, Chen T, Zhou HJ. 2010. Effect of Theabrownin in Pu-erh tea on the hormone-sensitive lipase activity and mRNA expression in rat[J]. Acta Nutr Sin, 32(4): 362-366. [高斌, 彭春秀, 龚 加顺, 陈婷, 周红杰. 2010. 普洱茶茶褐素对大鼠激素敏感性脂肪 酶活性及其 mRNA 表达的影响. 营养学报, 32(4): 362-366.]

Gong JS, Hu XJ, Peng CX, Zhou HJ. 2010. The molecular composition and spectral properties of polysaccharide isolated from Pu-erh Tea and its material[J]. Spectrosc Spect Anal, 30(7): 1960-1964. [龚加顺, 胡小静, 彭春秀, 周红杰. 2010. 普洱茶及其原料多糖分子组成及光谱学特 性研究. 光谱学与光谱分析, 30(7): 1960-1964.]

Gong JS, Peng CX, Chen T, Gao B, Zhou HJ. 2010. Effects of theabrownin from Pu-erh tea on the metabolism of serum lipids in rats: mechanism of action[J]. J Food Sci, 75(6): H182-H189.

Gu R, Li RM, Zhang LL, Zhou SP, Liang JY. 2011. Advances in study on
500 700 个脂肪储存调控基因。这些脂代谢和调控 基因中，哪些基因的表达或者蛋白水平和酶活性受 普洱茶调控，最终起到减肥、降脂作用？另外，胰 岛素/IGF-1 信号通路和 mTOR 信号通路等一些重要 的能量代谢信号通路是否也受普洱茶的影响呢?

近 10 年来, 转录组学、蛋白组学和脂质组学发 展迅速，这些新方法和新技术为了解疾病发病机 理、寻找疾病标记物和药靶提供了强有力的手段。 因此, 未来可以广泛地开展调查研究普洱茶与脂代 谢和调控基因的表达或相关酶活性，以及多条调控 能量代谢信号通路之间的关系; 利用上述的组学技 术和方法寻找新的普洱茶作用靶基因。总之，目前 对普洱茶减肥、降脂作用机理的研究才刚刚起步, 未来需要从多方面、多角度深入探索和阐明普洱茶 的减肥、降脂机制。

chemical composition and pharmacological properties of Pu-erh tea[J]. Tianjin Pharm, 23(1): 47-50. [顾睿, 李瑞明, 张兰兰, 周水平, 梁敬 钜. 2011. 普洱茶化学成分及药理研究进展. 天津药学, 23(1): 47-50].

Hou Y, Shao WF, Xiao R, Xu KL, Ma ZZ, Johnstone BH, Du YS. 2009 $\mathrm{Pu}$-erh tea aqueous extracts lower atherosclerotic risk factors in a rat hyperlipidemia model[J]. Exp Gerontol, 44(6-7): 434-439.

Hou YF, Wang DF, Zhou XL, Zhang L, Wang JF, Bucheli P. 2008. Effects of tea polysaccharides on blood lipids and liver trace elements in Hyperlipidemic rats[J]. Acta Nutr Sin, 30(3): 269-272. [侯仰锋, 汪东 风, 周小玲, 张莉, 王静风, Bucheli P. 2008. 茶多糖对高脂血症大 鼠血脂及肝脏中微量元素的调节作用. 营养学报, 30(3): 269-272.]

Huang HC, Lin JK. 2011. Pu-erh tea, green tea, and black tea suppresses hyperlipidemia, hyperleptinemia and fatty acid synthase through activating AMPK in rats fed a high-fructose $\operatorname{diet}[\mathrm{J}]$. Food Funct, 3(2): 170-177.

Huang J, Hao Y, Tao X, Rao XQ, Huang Z, Qi KB. 2010. The Mechanisms of Weight-cutting Effect in Pu-erh Tea[J]. Guangdong Tea, (4): 34-37. [黄建, 郝洋, 陶金金, 饶兴蓄, 黄智, 戚康标. 2010. 普洱茶减肥机制 的探讨. 广东茶业, (4): 34-37.]

Jeng KC, Chen CS, Fang YP, Hou RC, Chen YS. 2007. Effect of microbial fermentation on content of statin, GABA, and polyphenols in Pu-erh tea[J]. J Agric Food Chem, 55(21): 8787-8792.

Jiang XF, Shao WF. 2011. Effect of Pu-erh tea on blood lipid and plasma TXB2 and 6-Keto-PGF1 $\alpha$ levels in Hyperlipidemic mice[J]. Food Sci, 32(17): 317-320. [江新风, 邵宛芳. 2011. 普洱茶对高脂血症大鼠血 脂、血浆中血栓 B2、6-酮前列腺素 $F 1 \alpha$ 水平影响. 食品科学, 32(17): 317-320.]

Ku KM, Kim J, Park HJ, Liu KH, Lee CH. 2010. Application of metabolomics in the analysis of manufacturing type of pu-erh tea and composition changes with different postfermentation year[J]. J Agric Food Chem, 58(1): 345-352.

Kuo KL, Weng MS, Chiang CT, Tsai YJ, Lin-Shiau SY, Lin JK. 2005. Comparative studies on the hypolipidemic and growth suppressive effects of oolong, black, pu-erh, and green tea leaves in rats[J]. $J$ Agric Food Chem, 53(2): 480-489. 
Li J, Qiu X, Fan P, Chang C, Yang CR, Zhang YJ, Chen GZ, Hu JB. 2009. Sixty cases of Pu-erh tea pills adjusting hyperlipidemia[J]. Chn Med Mod Dis Educat China, 7(11): 22-23. [李捷, 邱湘, 范萍, 常畅, 杨崇 仁, 张颖君, 陈国珍, 胡金波. 2009. 普洱茶片调节高脂血症 60 例. 中国中医药现代远程教育, 7(11): 22-23.]

Lu HP, Lin Z, Gu JP, Guo L, Tan JF. 2007. Study on the gallic acid in Pu-erh tea[J]. J Tea Sci, 27(2): 104-110. [吕海鹏, 林智, 谷记平, 郭丽, 谭俊 峰. 2007. 普洱茶中的没食子酸研究. 茶叶科学, 27(2): 104-110.]

Lu JH, Sun LX. 2006. Investigation of Pu-erh tea active principles to inhibit the cholesterol synthesis in Hep G2 cell line[C] // The Fourth Cross-Straits Tea Industry Proceedings. [吕季桦, 孙璐西. 2006. 普洱 茶抑制 HepG2 细胞株生合成胆固醇之有效成分探讨 // 第四届海峡 两岸茶业学术研讨会论文集.]

Luo LX, Wu XC, Deng YL, Fu SW. 1988. Variations of main biochemical components and their relations to quality formation during pile-fermentation process of Yunnan Puer tea[J]. J Tea Sci, 18(1): 53-60. [罗龙新, 吴小崇, 邓余良, 傅尚文. 1988. 云南普洱茶渥堆过程中 生化成分的变化及其与品质形成的关系. 茶叶科学, 18(1): 53-60.]

Ow YY, Stupans I. 2003. Gallic acid and gallic acid derivatives: effects on drug metabolizing enzymes[J]. Curr Drug Metab, 4(3): 241-248.

Qin JH, Li N, Tu PF, Ma ZZ, Zhang L. 2012. Change in tea polyphenol and purine alkaloid composition during solid-state fungal fermentation of postfermented tea[J]. J Agric Food Chem, 60(5): 1213-1217.

Sano M, Takenaka Y, Kojima R, Saito S, Tomita I, Katou M, Shibuya S. 1986. Effects of Pu-erh tea on lipid metabolism in rats[J]. Chem Pharm Bull, 34(1): 221-228.

Shao CF, Jia LH, Li CW, Wei JP, Liu SH. 2011. The theabrownin in Pu-erh tea research progress[J]. Tianjin Chem Ind, 25(6): 1-3. [邵春甫, 贾黎 晖, 李长文, 魏纪平, 刘顺航. 2011. 普洱茶茶褐素研究进展. 天津 化工, 25(6): 1-3.]

Syu KY, Lin CL, Huang HC, Lin JK. 2008. Determination of theanine, GABA, and other amino acids in green, oolong, black, and Pu-erh teas with dabsylation and high-performance liquid chromatography[J]. $J$ Agric Food Chem, 56(17): 7637-7643.

Turnbaugh PJ, Gordon JI. 2009. The core gut microbiome, energy balance and obesity[J]. J Physiol, 587(Pt 17): 4153-4158.

Wang Q,P Peng CX, Gong JS. 2011. Effects of enzymatic action on the formation of theabrownin during solid state fermentation of Pu-erh tea[J]. J Sci Food Agric, 91(13): 2412-2418.

Way TD, Lin HY, Kuo DH, Tsai SJ, Shieh JC, Wu JC, Lee MR, Lin JK. 2009. Pu-erh tea attenuates hyperlipogenesis and induces hepatoma cells growth arrest through activating AMP-activated protein kinase (AMPK) in human HepG2 cells[J]. J Agric Food Chem, 57(12): 5257-5264.

Wu WH. 2005. Sun-dried Tea (Maocha) and Pu-erh tea hypolipidemic effect comparison test[J]. China Tea, (1): 15. [吴文华. 2005. 晒青毛茶和普 洱茶降血脂作用比较试验. 中国茶叶, (1): 15.]
Wu WH, Wu WJ. 2006. The Pu-erh tea polysaccharide lipid-lowering function dose-effect relationship[J]. Tea in Fujian, (2): 42-43. [吴文华, 吴文俊. 2006. 普洱茶多糖降血脂功能的量效关系. 福建茶叶, (2): 42-43.]

Wu WJ, Wu WZ, Wu WH. 2007. The polyphenols and polysaccharide in Pu-erh tea lipid -lowering functional comparison[J]. Tea in Fujian, (3): 42-43. [吴文俊, 吴镇洲 吴文华. 2007. 普洱茶中茶多糖与茶多酚 降血脂功能比较. 福建茶叶, (3): 42-43.]

Xie GX, Ye M, Wang YG, Ni Y, Su MM, Huang H, Qiu MF, Zhao AH, Zheng XJ, Chen TL, Jia W. 2009. Characterization of pu-erh tea using chemical and metabolic profiling approaches[J]. J Agric Food Chem, 57(8): 3046-3054.

Xu XT, Wang P, Luo SZ, Luo Y, Jiang XF, Hou Y, Shao WF, Yang JH. 2011. Effects of Pu-erh tea on hyperlipidemia prevention, antioxidation and vascular endothelium protection in SD rats[J]. J Yunnan Agri Univ, 26(2): 260-264. [徐湘婷, 王鹏, 罗绍忠, 罗渏, 江新凤, 侯艳, 邵宛 芳, 杨继红. 2011. 普洱茶预防 SD 大鼠高脂血症及抗氧化、保护血. 管内皮的研究. 云南农业大学学报, 26(2): 260-264.]

Yang DJ, Hwang LS, Lin JT. 2007. Effects of different steeping methods and storage on caffeine, catechins and gallic acid in bag tea infusions[J]. J Chromatogr A, 1156(1-2): 312-320.

Yang TTC, Koo MWL. 1997. Hypocholesterolemic effects of Chinese tea[J] Pharmacol Res, 35(6): 505-512.

Yuan HB, Zhong J, Yi J, Zhao Y, Cao J. 2009. Effect of Pu-erh tea on lipogenesis and expression of relative genes in obese rat fed with high fat diet[J]. Acta Nutr Sin, 31(2): 167-171，176. [袁华兵，钟婕，易娟， 赵燕, 曹进. 2009. 普洱茶提取物对饮食诱导肥胖大鼠脂质合成相 关基因表达的影响. 营养学报, 31(2): 167-171, 176.]

Zhang DY, Shao WF, Liu ZH, Liu YL, Huang YW. 2009. Research on the Anti-diabetes and Anti-hyperlipidenmia function of monomers in Pu-erh tea[J]. J Tea Sci, 29(1): 41-46. [张冬英, 邵宛芳, 刘仲华, 刘 亚林, 黄业伟. 2009. 普洱茶功能成分单体降糖降脂作用研究. 茶 叶科学, 29(1): 41-46.]

Zhang L, Li N, Ma ZZ, Tu PF. 2011. Comparison of the chemical constituents of aged Pu-erh tea, ripened pu-erh tea, and other teas using HPLC-DAD-ESI-MSn[J]. J Agric Food Chem, 59(16): 8754-8760.

Zhao LP, Shao WF. 2010. Effects of Pu-erh tea on blood lipid and fatty liver in rats[J]. Southwest China J Agric Sci, 23(2): 579-583. [赵丽萍, 邵宛 芳. 2010. 普洱茶对高脂血症大鼠的降脂和预防脂肪肝作用. 西南 农业学报, 23(2): 579-583.]

Zhou NN, Dai R, Li SM, He XS, Lin Q. 2009. Study on the pharmacological effect of Pu-erh tea[J]. Chn Arch Trad Chn Med, 27(7): 1535-1536. [周宁娜, 代蓉, 李松梅, 何晓山, 林青. 2009. 普洱茶减 肥作用的药理学基础研究. 中华中医药学刊, 27(7): 1535-1536.]

Zuo YG, Chen H, Deng YW. 2002. Simultaneous determination of catechins, caffeine and gallic acids in green, Oolong, black and Pu-erh teas using HPLC with a photodiode array detector[J]. Talanta, 57(2): 307-316. 